l' $A M P$ cyclique entraînait la translocation dans le noyau de la seule sous-unité $C, R_{\mathrm{II}}$ restant dans le cytoplasme. Ces résultats furent en fait vivement controversés durant la réunion de Nem York. Plus important, les laboratoires de Richard Hanson (Cleveland, USA) et de Gunther Schütz (Heidelberg, RFA) ont déterminé par différentes méthodes quelle était la séquence d'ADN en amont des gènes de la phosphoenolpyruvate carboxykinase et de la tyrosine aminotransférase qui semblait essentielle à l'action de l'AMP cyclique. Or ce n'est pas préférentiellement à ce niveau que semble se fixer $R_{\mathrm{II}}$. Il n'empêche que la découverte de fungman ouvre une voie nouvelle de recherche, d'autant plus intéres- sante que des résultats similaires ont été présentés récemment par Miguel Beato (Marburg, RFA) concernant l'action du récepteur des glucocorticoïdes liés à l'hormone (Réunion des sociétés allemandes, françaises et suisses de Biochimie, Bâle, octobre I985).

A. $\mathrm{K}$.

I. Munnich A, Vaulont S, Marie J. De nouvelles fonctions pour l'AMP cyclique. médecine-sciences 1985; 1 : $192-17$.

2. Constantinou A L, Squinto SP, Jungmann RA. The phosphoform of the regulatory subunit $R$ II of cyclic AMP-dependent protein kinase possesses intrinsic topoisomerase activity. Cell $1985 ; 42: 429-37$.

3. Nigg EA, Hilz H, Eppenberger HM, Dutly F Rapid and reversible translocation of the catalytic subunit of cAMP-dependent protein kinase type II from the Golgi complex to the nucleus. Embo 7 1985; 4 : 280I-6.

\title{
La trans-activation du virus LAV est traductionnel/e
}

La synthèse des protéines virales dans une cellule lymphocytaire infectée par le virus LAV responsable du SIDA, est considérablement stimulée par le produit d'un des gènes viraux, le gène Tat III. Le facteur trans-activateur * codé par Tat III reconnaît une courte séquence nucléotidique dénommée TAR (Trans-Activating Responsive sequence) qui est localisée dans le LTR (Long Terminal Repeat) du LAV. Un génome viral délété en gène Tat III synthétise de 500 à 2000 fois plus de produits viraux dans une cellule produisant de façon constitutive (c'est-à-dire permanente) le facteur Tat III que dans

\footnotetext{
* Une activation "en trans " se fait à distance du fragment d $A D N$ qui en est responsable, par opposition à une activation "en cis "qui ne s'exerce que sur un gène contigu au fragment activateur. Un " transactivateur " est un facteur diffusible, produit du gène activateur.

$\mathrm{m} / \mathrm{s} n^{0}$ 5, vol. 2, mai 86
}

une cellule normale. Ce mécanisme de trans-activation également noté pour les virus HTLV et de nombreux virus à $\mathrm{ADN}$, est extrêmement important pour expliquer l'abondante production virale par les cellules infectées et donc en partie le pouvoir pathogène. Rosen et al. du laboratoire de W. Haseltine à Boston (USA), viennent néanmoins de démontrer que les concentrations d'ARN viral, ou de l'ARN d'un gène placé sous le contrôle du LTR du LAV, étaient identiques dans des cellules exprimant ou non le facteur trans-activateur [I].

Afin de déterminer les séquences du LTR viral indispensables au phénomène de trans-activation, les auteurs construisirent plusieurs gènes hybrides comportant, en differentes positions, des fragments du LTR viral. La séquence située entre les bases +38 et +80 (la position $+\mathrm{I}$ définit le site d'initiation de la trans- 
cription du génome viral) est active quand elle est insérée en aval du site d'initiation de la transcription du gène hybride et se retrouve à l'extrémité 5' de l'ARN transcrit.

L'explication la plus simple de tous ces résultats est que le facteur Tat III interfère avec l'extrémité $5^{\prime}$ du messager et en augmente considérablement la traductibilité. Nous nous trouvons donc là encore, comme il est signalé dans une autre nouvelle de ce numéro, devant une régulation non transcriptionnelle de l'expression d'un gène.

Des substances antibiotiques qui interferrent avec la traduction des mes- sagers pourraient ainsi inhiber le phénomène de trans-activation du virus $L A V$, et constituent donc une intéressante voie de recherche thérapeutique.

Dans un tout autre domaine, l'utilisation de la séquence TAR et du produit Tat III pourrait constituer un moyen efficace d'augmenter considérablement la production par génie génétique de substances d'intérêt biologique.

A.K.

I. Rosen CA, Sodroski JG, Goh WH, et al. Posttranscriptional regulation accounts for the transactivation of the human $\mathrm{T}$-lymphotropic virus type III. Nature 1986; $319: 555-9$.

\section{Régulation post-transcriptionnelle de l'expression des oncogènes}

Le Lexique du $n^{\circ} 7$ vol. I de médecine/sciences a déjà insisté sur l'importance de la régulation de l'expression des gènes après leur transcription en $A R N$. Cette régulation peut intéresser la maturation des transcrits, la stabilité des messagers ou leur traductibilité. Or, depuis les travaux réellement novateurs $d u$ laboratoire de Philippe Feanteur à Montpellier, il apparaît que c'est en effet à des niveaux posttranscriptionnels qu'est régulée l'expression de plusieurs oncogènes. Le messager de l'oncogène c-myc a une durée de vie extrêmement brève (demi-vie de l'ordre de 10 minutes). Lorsqu'une cellule quiescente est stimulée par un facteur de croissance, l'augmentation de la concentration $d u$ messager c-myc est un phénomène précoce qui n'est pas d̂̀ à une augmentation de sa synthèse, mais à sa stabilisation provisoire $\left[\begin{array}{ll}I, & 2\end{array}\right]$. L'interféron réprime l'expression de l'ARN c-myc, et donc de la protéine correspondante, en le déstabilisant [3]. Dans des plasmocytomes de souris aussi bien que dans certains lymphomes de Burkitt, il existe des remaniements chromosomiques qui, très souvent, séparent le premier exon non codant du gène des deux exons codants. L'ARN transcrit est alors tronqué. Deux équipes différentes viennent de démontrer que l'accumulation de ces messagers aberrants dans ces cellules tumorales était secondaire à une augmentation de la stabilité de l'ARN c-myc dépourvu du premier exon [4-6].

Un tel phénomène d'une régulation post-transcriptionnelle au cours $d u$ cycle cellulaire a été rapporté également à propos de deux autres oncogènes dont les produits ont une localisation nucléaire : p53 (oncogène cellulaire codant pour une protéine de 53000 de poids moléculaire et n'ayant pas d'équivalent viral [7]) et c-myb [8]. Lorsqu'une cellule quiescente est stimulée à proliférer, un produit d'oncogène augmente encore plus précocement que c-myc: il s'agit de c-fos dont le messager est, comme celui de c-myc, très instable. $L a$ dégradation rapide de ce messager après stimulation requiert l'intégrité de la séquence $3^{\prime}$ non codante; lorsqu'elle est délétée, la concentration de l'ARN c-fos reste élevée après stimulation et un gène ainsi modifié devient transformant par transfection dans des cellules fibroblastiques [9]. La conclusion de toutes ces études est que la concentration intracellulaire des produits d'oncogènes cellulaires, et donc aussi leur pouvoir transformant, est fréquemment régulée au niveau de la stabilité de leurs messagers, ce qui ne signifie pas que des phénomènes transcriptionnels ne puissent pas également intervenir. Des séquences de l' $A R N$, situées dans la région $5^{\prime}$ non codante dans le cas de c-myc et $3^{\prime}$ non codante dans le cas de c-fos, jouent un grand rôle dans cette aptitude des $A R N$ à être régulés par leur dégradabilité. On peut imaginer que ces segments conferent aux transcrits une conformation particulière, les exposant à la dégradation sous l' influence de facteurs régulés au cours du cycle cellulaire et de l'induction de la prolifération.

A.K.

I. Dani C, Blanchard J M, Piechaczyk M, et al. Extreme instability of myc mRNA in normal and transformed human cells. Proc Natl Acad $\mathrm{Sci}_{\mathrm{i}}$ $U S A$ 1984; 81: 7046-50.

2. Blanchard J M, Piechaczyk M, Dani C, et al. $c$-myc gene is transcribed at high rate in $\mathrm{G}_{0}$ arrested fibroblasts and is post-transcriptionnally regulated in response to growth factors. Nature 1985; $317: 443-5$.

3. Dani C, Mechti N, Piechaczyk M, et al. Increased rate of degradation of $c$-myc mRNA in interferon treated Daudi cells. Proc Natl Acad Sci $U S A$ 1985; $82: 4896-9$.

4. Eick D, Piechaczyk M, Henglein B, et al. Aberrant c-myc RNAs of Burkitt's lymphoma cells have longer half lives. Embo 7 1985; 4 : 3717-25.

5. Piechaczyk M, Yang J Q Blanchard J M et al. Posttranscriptional mechanisms are responsible for accumulation of truncated c-myc RNAs in murine plasma cell tumors. Cell $1985 ; 42: 589-$ 97.

6. Rabbitts P H, Forster A, Stinson M A et al. Truncation of exon I of the $c$-myc gene results in prolonged $c$-myc mRNA stability. Embo 7 ig85; $4: 3727-33$.

7. Dony C, Kessel M, Gruss, P. Posttranscriptional control of $c$-myc and p53 expression during differentiation of the embryonal carcinoma cell line F9. Nature $1985 ; 317: 636-8$.

8. Thompson C B, Challoner P B, Weiman P E, et al. Expression of the $c$-myb proto-oncogene during cellular proliferation. Nature 1986; 319: 374-8o.

9. Treisman R. Transient accumulation of $c$-fos RNA following serum stimulation requires a conserved $5^{\prime}$ element and $c$-fos $3^{\prime}$ sequences. Cell I985; $42: 889-902$. 